\title{
Quasi-Elliptic Ultra-Wideband Bandpass Filter with Super-Wide Stopband
}

\author{
Muhammad Riaz, Bal S. Virdee*, Panchamkumar Shukla, and Muhittin Onadim \\ London Metropolitan University, Centre for Communications Technology, 166-220 Holloway Road, London, UK. \\ "b.virdee@londonmet.ac.uk
}

\begin{abstract}
This paper describes a low-loss and compact planar ultra-wideband (UWB) quasi-elliptical bandpass filter, which is based on a via-free multimode resonator (MMR) that requires no defected ground plane. The MMR structure essentially consists of a step impedance transmission-line resonator that is loaded centrally with a folded open-circuited stub. The MMR structure is also loaded with two pairs of short high impedance stubs with their ends folded towards the central stub. The proposed MMR structure excites several resonant modes comprising three even and two oddmodes within the filter's passband. The resulting transmission response is highly selective with a low-loss (0.4 $\mathrm{dB})$ passband across $3.60 \mathrm{GHz}-11.3 \mathrm{GHz}$ when fabricated on a conventional dielectric substrate, which is otherwise only possible using high temperature superconductors. In addition, the structure generates transmission zeros to yield a filter that exhibits an extremely wide stopband extending up to $35.5 \mathrm{GHz}$ for $\mathrm{S}_{21} \geq 20 \mathrm{~dB}$. The via-free planar filter structure makes its design and construction easy and economical for mass production. The filter's performance was verified through measurements. The planar filter structure can be easily manufactured using standard PCB technology.
\end{abstract}

\section{Introduction}

Being a vital component of multi-standard wireless communication systems, ultra-wideband (UWB) bandpass filters are receiving great attention by researchers in industry and academia ever since the Federal Communications Commission (FCC) authorized the unlicensed use of the frequency band between 3.1 to $10.6 \mathrm{GHz}$ for commercial use [1]. Over the recent years there has been a tremendous increase in the development of various UWB filter structures using various techniques. These devices have been employed in highspeed mobile local area networks, ground penetrating and through-wall radar, medical imaging, secure communications etc. [2]. Although traditional filter designs based on microstrip technology possess desirable features of low loss, high returnloss and light weight, however these filters do not meet the stringent requirements of next generation wireless systems including sharp roll-off, wide rejection stopband and flat passband group delay [2]-[4]. Makimoto in [5] first proposed the use of stepped impedance resonators (SIR) in the design of bandpass filters (BPF) to address issues that degrade filter performance in terms of parasitic bands and narrow stopband between the first spurious response and the fundamental mode. These issues cannot be eradicated using traditional filters based on quarter or half wavelength parallel coupled resonators. The impedance ratio of SIR can be used to control the parasitic band and spurious responses without enlarging the overall circuit size. Currently different methods are being used to develop various types of filter structures including multimode resonators (MMR) [6]. This concept was initially used to miniaturize the UWB filter structure in [7] where three resonant modes of multimode resonator were used to realize the filter.

In this paper, a compact ultra-wideband planar bandpass filter is described that exhibits desirable characteristics of a sharp quasi-elliptical response, low passband loss, high selectivity and an extremely wide out-of-band rejection reported to date [8]-[20]. These features are normally achieved using high temperature superconductors. Unlike other UWB filter designs the proposed filter structure requires no shortcircuited vias and defected ground plane. The design of the proposed filter is based on MMR configuration consisting of a stepped impedance transmission-line resonator loaded with four bent open-circuited stubs that form a split-ring square resonator. The transmission-line resonator is also loaded centrally with a stepped impedance stub. The MMR is interdigitally coupled to the input and output feedlines to realize a strong electromagnetic interaction. The proposed MMR excites three even and two odd-mode resonances in the passband. The fabricated filter was practically characterized to verify its performance.

\section{Analysis of the Proposed SLR}

The proposed multimode resonator is based on the MMR structure in [21]. The MMR configuration constituting the proposed UWB filter is essentially a stepped impedance transmission-line resonator loaded with a centrally located stepped impedance open-circuited stub, which is folded to minimize the overall space occupied by the filter, as shown in Fig. 1. Wide out-of-band rejection is implemented by loading the basic MMR structure with two sets of parallel connected high impedance open-circuit stubs that are inwardly folded. The two-parallel open-circuit stubs have admittance and length defined by $Y_{01}$ and $\theta_{S}$, in Fig. 2. These two stubs create finite transmission zeros determined by their lengths. The MMR structure is electromagnetically coupled to the input and output through interdigital capacitance. The characteristic admittance 
and length of the horizontal stepped impedance transmissionline resonator are $Y_{1}, \theta_{l}$, and $Y_{2}, \theta_{2}$, respectively; and the characteristic admittance and electrical length of the central stepped impedance stub are $Y_{3}, \theta_{3}$, and $Y_{4}, \theta_{4}$, respectively, as depicted in Fig. 2. Since the MMR is symmetrical about B-B' plane it can be analyzed using odd and even-mode analysis. Fig. 3(a) and 3(b) show the even- and odd-mode circuits. Under oddmode excitation there is a voltage null in the middle of the MMR structure. This leads to the equivalent circuit of Fig. 3(b).

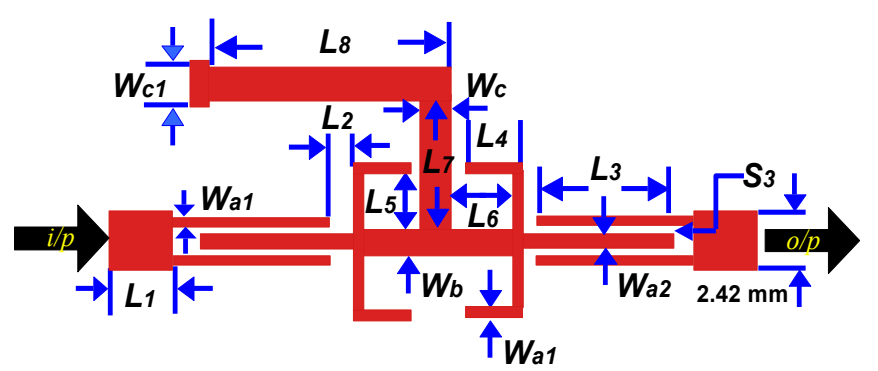

Fig. 1. Structure of the proposed UWB quasi-elliptical bandpass filter.



Fig. 2. Configuration of basic MMR structure. Parallel opencircuit stubs are indicated in dotted lines.

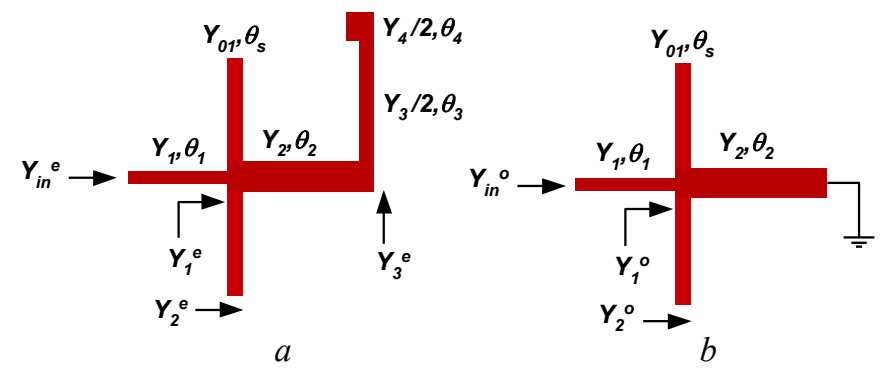

Fig. 3. Equivalent circuit model of the MMR structure (a) even-mode circuit, (b) odd-mode circuit model.

The admittances in the even-mode structure are represented by

$Y_{i n}^{e}=Y_{1}\left[\frac{Y_{1}^{e}+j Y_{1} \tan \theta_{1}}{Y_{1}+j Y_{1}^{e} \tan \theta_{1}}\right]$

where

$Y_{1}^{e}=Y_{01}\left[\frac{Y_{2}^{e}+j Y_{01} \tan \theta_{s}}{Y_{01}+j Y_{2}^{e} \tan \theta_{S}}\right]$
$Y_{2}^{e}=Y_{2}\left[\frac{Y_{3}^{e}+j Y_{2} \tan \theta_{2}}{Y_{2}+j Y_{3}^{e} \tan \theta_{2}}\right]$

$Y_{3}^{e}=j \frac{Y_{3}}{2}\left[\frac{Y_{4} \tan \theta_{4}+Y_{3} \tan \theta_{3}}{Y_{3}-Y_{4} \tan \theta_{3} \tan \theta_{4}}\right]$

Admittances in the odd-mode structure are represented by

$Y_{i n}^{o}=Y_{1}\left[\frac{Y_{1}^{o}+j Y_{1} \tan \theta_{1}}{Y_{1}+j Y_{1}^{o} \tan \theta_{1}}\right]$

where

$Y_{1}^{o}=Y_{01}\left[\frac{Y_{2}^{o}+j Y_{01} \tan \theta_{s}}{Y_{01}+j Y_{2}^{o} \tan \theta_{S}}\right]$

$Y_{2}^{o}=-j Y_{2} \cot \theta_{2}$

At resonance, $Y_{i n}{ }^{e}=0$ and Eqn. (1) simplifies to

$Y_{1}^{e}=-j Y_{1} \tan \theta_{1} \quad$ or $\quad Y_{01}\left[\frac{Y_{2}^{e}+j Y_{01} \tan \theta_{s}}{Y_{01}+j Y_{2}^{e} \tan \theta_{S}}\right]=-j Y_{1} \tan \theta_{1}$

$Y_{2}^{e}=-Y_{01}\left[\frac{Y_{1} \tan \theta_{1}+Y_{01} \tan \theta_{s}}{Y_{01}-Y_{1} \tan \theta_{1} \tan \theta_{S}}\right]=Y_{2}\left[\frac{Y_{3}^{e}+j Y_{2} \tan \theta_{2}}{Y_{2}+j Y_{3}^{e} \tan \theta_{2}}\right]$

or $-Y_{01}\left(Y_{1} \tan \theta_{1}+Y_{01} \tan \theta_{s}\right)\left(Y_{2}+j Y_{3}^{e} \tan \theta_{2}\right)=$ $Y_{2}\left(Y_{3}^{e}+j Y_{2} \tan \theta_{2}\right)\left(Y_{01}-Y_{1} \tan \theta_{1} \tan \theta_{s}\right)$

Similarly, for the odd-mode structure at resonance $Y_{i n}{ }^{o}=0$ and Eqn. (2) simplifies to

$$
Y_{01}\left(Y_{1} \tan \theta_{1}+Y_{01} \tan \theta_{s}\right)=Y_{2} \cot \theta_{2}\left(Y_{01}-Y_{1} \tan \theta_{1} \tan \theta_{s}\right)
$$

Even-mode resonance frequencies can then be deduced from Eqn.(3), i.e. when $\tan \theta_{1} \tan \theta_{s}=1 / k_{1}$ and $k_{1} \tan \theta_{1}=\tan \theta_{s}$, where $k_{1}=Y_{1} / Y_{01}$. For simplicity, when electrical length $\theta_{1}=\theta_{s}=\theta$, where $\theta=\beta l$ the even-mode resonance frequencies occur at $\theta\left(f_{1}\right)=\pi, \theta\left(f_{2}\right)=\tan ^{-1} \sqrt{1 / k_{1}}$, $\theta\left(f_{3}\right)=\pi-\tan ^{-1} \sqrt{1 / k_{1}}, \theta\left(f_{4}\right)=\tan ^{-1} \sqrt{k_{3}}$, and $\theta\left(f_{5}\right)=$ $\pi-\tan ^{-1} \sqrt{k_{3}}$, where $k_{3}=Y_{3} / Y_{4}$. Similarly, from Eqn.(4) when $\theta_{1}=\theta_{s}=\theta$ the odd-mode frequencies deduced are $\theta\left(f_{6}\right)=\pi / 2$, and the expressions are identical for $\theta\left(f_{2}\right)$ and $\theta\left(f_{3}\right)$.

The fundamental and higher order resonant frequencies can be adjusted over a wide frequency range by modifying the admittance ratio $(k)$ and the length ratio $\alpha$ defined as

$\alpha=\theta_{i} /\left(\theta_{i}+\theta_{i+2}\right) \quad$ where $i=1,2,3 \ldots$

Depending on the choice of $\alpha$ and $k$, it is feasible to couple different resonant modes to obtain a wide passband. If the microstrip lines are non-dispersive and line sections have equal phase velocity as $\alpha \propto f$, we obtain

$f_{1} / f_{6}=2$

$f_{2} / f_{6}=(2 / \pi) \tan ^{-1} \sqrt{1 / k_{1}}$

$f_{3} / f_{6}=(2 / \pi)\left(\pi-\tan ^{-1} \sqrt{1 / k_{1}}\right)$

$f_{4} / f_{6}=(2 / \pi) \tan ^{-1} \sqrt{k_{3}}$

$f_{5} / f_{6}=(2 / \pi)\left(\pi-\tan ^{-1} \sqrt{k_{3}}\right)$ 
Normalized separation relative to $f_{6}$ is given by:

$$
\begin{aligned}
& \Delta f_{32}=(2 / \pi)\left(\pi-2 \tan ^{-1} \sqrt{1 / k_{1}}\right) \\
& \Delta f_{54}=(2 / \pi)\left(\pi-2 \tan ^{-1} \sqrt{k_{3}}\right) \\
& \Delta f_{34}=(2 / \pi)\left(\tan ^{-1} \sqrt{1 / k_{1}}-\tan ^{-1} \sqrt{k_{3}}\right) \\
& \Delta f_{35}=(2 / \pi)\left(\tan ^{-1} \sqrt{k_{3}}-\sqrt{1 / k_{1}}\right)
\end{aligned}
$$

The above analysis shows the structure generates several resonance frequencies that create the wide passband of the filter with $f_{6}$ being the mid-band frequency, and where the separation equations determine the bandwidth. Frequency $f_{l}$ is always twice the mid-band frequency and is responsible for generating a higher spurious passband. The variation of various frequency parameters with change in the impedance ratio $(k)$ is depicted in Fig. 4, where $k=k_{1}=k_{3}$. The graph shows that when the impedance ratio is reduced, the normalized frequency $f_{2} / f_{6}$ increases and $f_{3} / f_{6}$ decreases. Thus, their trajectories move towards convergence. However, when $k$ is increased the normalized frequency $f_{4} / f_{6}$ tends to increase and $f_{5} / f_{6}$ tends to decrease so that their response tends towards convergence. This analysis shows by appropriately choosing $k$ the filter's 3 $\mathrm{dB}$ passband can be precisely controlled, i.e. either widened or narrowed. The Fig. 5 shows increase in separation is observed between the resonant mode frequency for mode 3 and 2, and 3 and 5; and the contrary is true for modes 3 and 4 , and 5 and 4 . The transmission zeros can be determined when $\mathrm{Y}_{i n}{ }^{e}=\mathrm{Y}_{i n}{ }^{o}$.

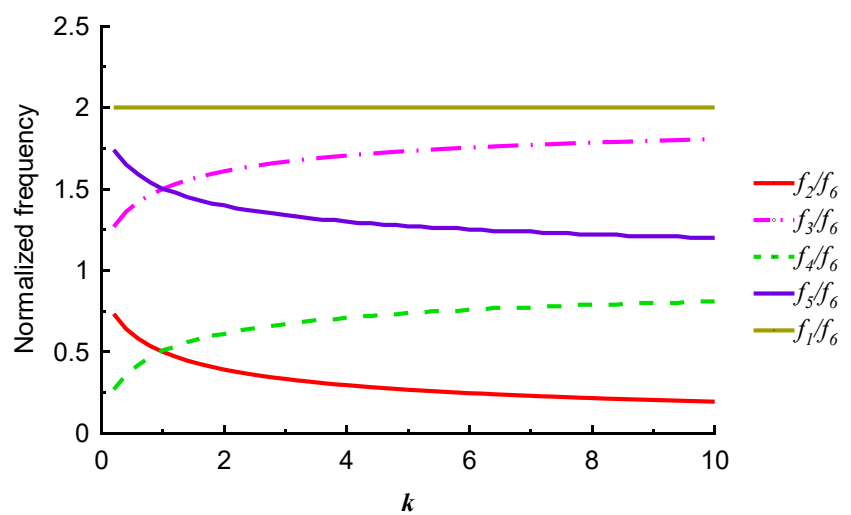

Fig. 4. Normalized frequency of resonance modes as a function of impedance ratio.

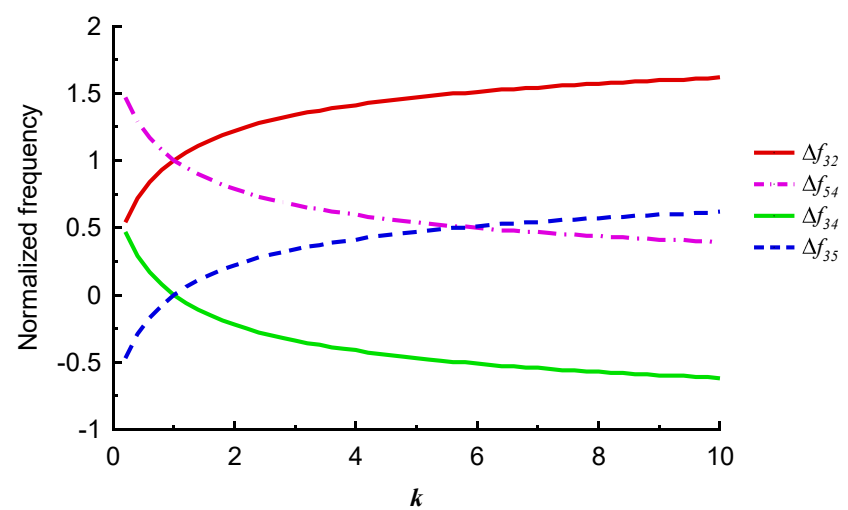

Fig. 5. Separation of the resonance mode frequencies as a function of impedance ratio.

\section{Parameter Analysis and Measured Results}

An ultra-wideband bandpass filter was synthesized using the proposed MMR structure shown in Fig. 1. The filter is excited through three finger interdigital capacitor to realize strong EM coupling. The filter was constructed on dielectric substrate Arlon CuClad217LX with thickness $(h)$ of $0.794 \mathrm{~mm}$, dielectric constant $\left(\varepsilon_{r}\right)$ of 2.17 , copper conductor thickness $(t)$ of $35 \mu \mathrm{m}$, and loss-tangent $(\tan \delta)$ of 0.0009 . To maintain good in-band performance, the physical dimensions were optimized by using Momentum in Advanced Design System (ADSTM). Optimised parameters are: $W_{b}=0.98 \mathrm{~mm}, W_{a}=0.19 \mathrm{~mm}, W_{c}=$ $1.41 \mathrm{~mm}, W_{c l}=1.96 \mathrm{~mm}, W_{a l}=0.18 \mathrm{~mm}, W_{a 2}=0.18 \mathrm{~mm}, L_{I}=$ $3 \mathrm{~mm}, L_{5}=1.51 \mathrm{~mm}, L_{6}=2.92 \mathrm{~mm}, L_{4}=0.39 \mathrm{~mm}, L_{7}=3.2$ $\mathrm{mm}, L_{8}=6.91 \mathrm{~mm}, L_{3}=5.92 \mathrm{~mm}, L_{2}=0.2 \mathrm{~mm}$, and $S_{3}=0.17$ $\mathrm{mm}$.

Effect of transmission-line resonator width $W_{b}$ on filter's performance was examined. When $W_{b}$ was varied from 0.2 to $1.0 \mathrm{~mm}$ it is shown in Fig. 6 the first odd-mode resonant frequency $f_{\text {oddl }}$ moves moderately upwards within the UWB passband to create a quintuple mode resonator, and the upper transmission zero $f_{t z 2}$ shifted slightly downwards in frequency. It should be noted that $W_{b}$ has virtually no impact on the other resonant modes and transmission zero frequencies.

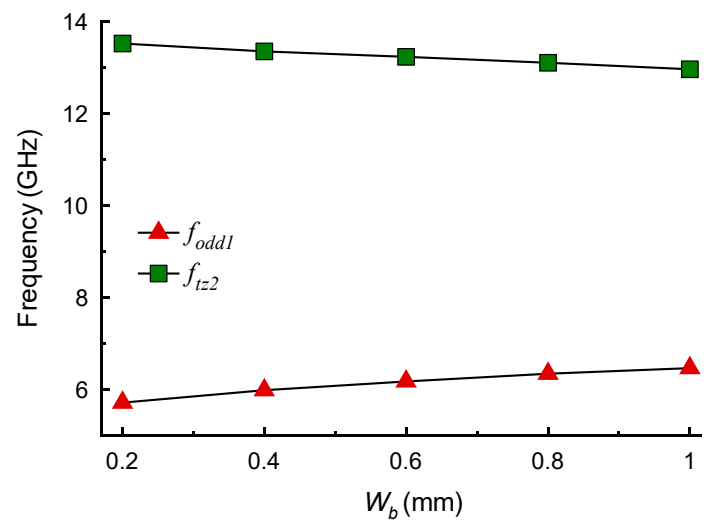

Fig. 6. Effect on the filter's upper transmission zero and first odd-mode resonant frequency as a function of resonator width $W_{b}$.

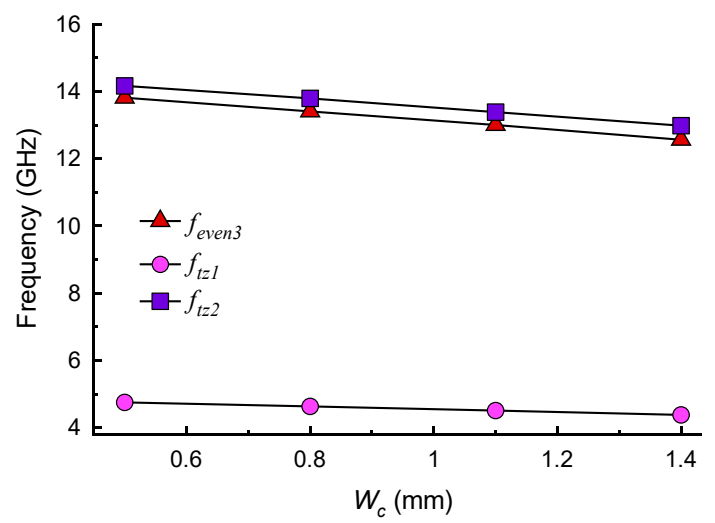

Fig. 7. Effect on the filter's lower and upper transmission zeros and third even-mode resonant frequency as a function of stub width $W_{c}$. 
Fig. 7 shows as the stub width $W_{c}$ is varied from $0.5 \mathrm{~mm}$ to $1.4 \mathrm{~mm}$, the resonant frequency of the third even-mode resonance $f_{\text {even } 3}$ and the upper transmission zero $f_{t z 2}$ move downwards in frequency, however the lower transmission zero $f_{t z l}$ shifts slightly downwards to create a wideband and sharp selectivity. This is because the length of the resonator is effectively increased and as the resonance frequency is inversely related to frequency the resonance frequency correspondingly decreases. Minor effect is also observed on the first and second odd-mode resonant frequencies and other resonant frequencies remain unaffected.

The effect of open-circuit stub length $L_{8}$ on the upper and lower transmission zero, and first and third even-mode resonant frequencies is shown in Fig. 8. It is evident that when $L_{8}$ is varied from $5.14 \mathrm{~mm}$ to $7 \mathrm{~mm}$, the upper transmission zero and third even-mode resonant frequency reduce in frequency more pronouncedly than the lower transmission zero and first even-mode resonant frequency. The open-circuit stub to some extent essentially acts like a notch function to create the upper and lower transmission zeros, i.e. attenuation, however it has negligible effect on resonant frequency $f_{\text {even } 2}$.

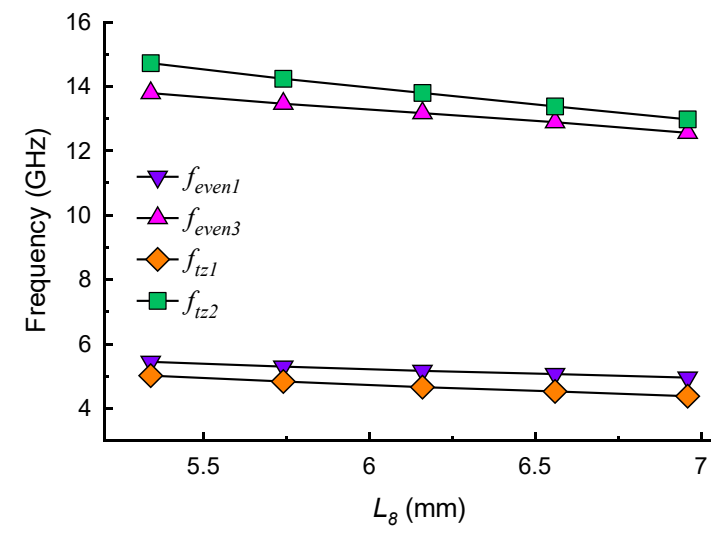

Fig. 8. Effect on the filter's lower and upper transmission zeros and first and third resonant mode frequencies as a function of stub length $L_{8}$.

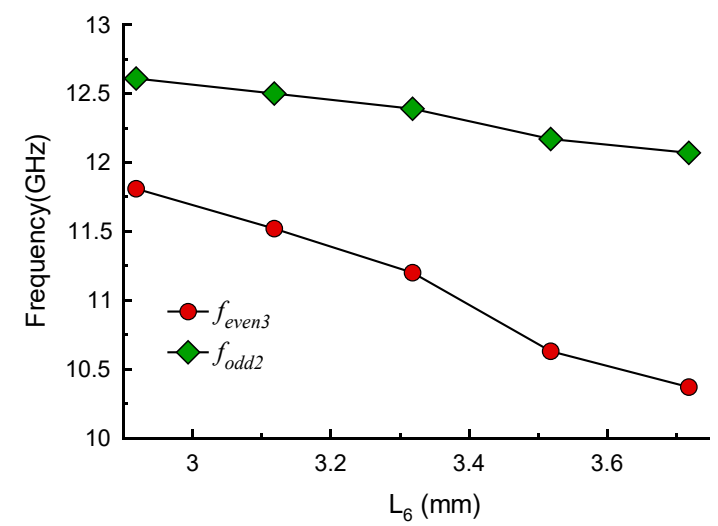

Fig. 9. Effect on the filter's second odd and third even-mode resonant frequencies as a function of length $L_{6}$.

Fig. 9 shows how the length $L_{6}$ controls the second odd-mode resonant frequency $f_{\text {odd } 2}$ and third even-mode resonant frequency $f_{\text {even } 3}$. As the length $L_{6}$ is increased from $2.92 \mathrm{~mm}$ to $3.72 \mathrm{~mm}$, the resonant frequency $f_{\text {even } 3}$ drops down more significantly than $f_{o 2}$. Length $L_{6}$ has no effect on the lower and upper transmission zero as well as on resonant frequencies $f_{\text {even } 2}$, $f_{\text {oddl } l}$ and $f_{\text {even } l}$.

Effect of the interdigital coupling length $L_{3}$ on the filter's characteristics is shown in Fig. 10. The frequency of resonant modes $f_{\text {oddl }}, f_{\text {odd } 2}$ and $f_{\text {even } 2}$ decrease as $L_{3}$ is increased from 4.34 $\mathrm{mm}$ to $5.94 \mathrm{~mm}$ Length $L_{3}$ has no effect on the transmission zeros and other resonant modes.

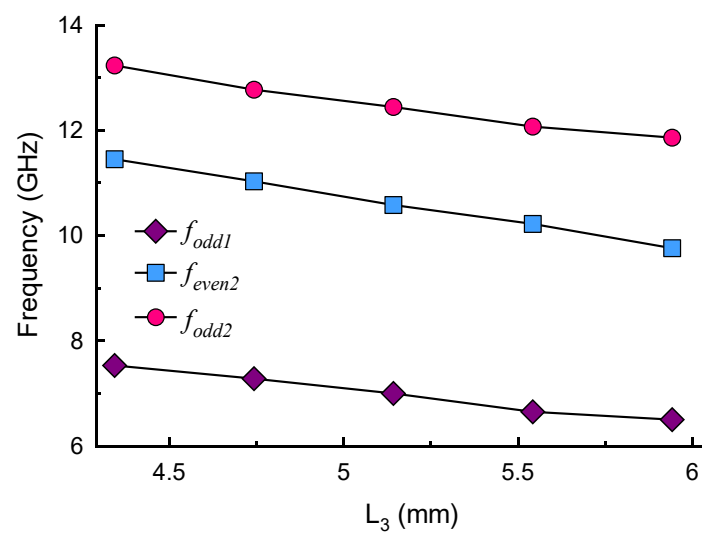

Fig. 10. Effect on the filter's first and second odd-mode and second even-mode resonant frequencies as a function of interdigital coupling length $L_{3}$.

The open stubs and the open-circuit coupled line, shown in Fig. 1, in the bandpass filter design generate multiple transmission zeros that contribute to establishing a wide stopband. Transmission zeros resulting from the open-stub lines are represented by $f_{z 1} \& f_{z 2}$, and the open-circuit coupled line by $f_{z 3}$. The frequency of transmission zeros are [22]:

$$
\begin{aligned}
f_{z 1} & =(2 n-1) f_{o} \frac{90^{o}}{\theta_{s}} \quad n=1,2, \ldots \\
f_{z 2} & =(2 n-1) f_{o} \frac{90^{o}}{\left(\theta_{3}+\theta_{4}\right)} \quad n=1,2, \ldots \\
f_{z 3} & =n f_{o} \frac{90^{\circ}}{\theta_{1}} \quad n=1,2, \ldots
\end{aligned}
$$

where $f_{o}$ is the centre frequency of the passband.

The fabricated MMR UWB bandpass filter is shown in Fig. 11. Measured performance of the proposed UWB bandpass filter is shown in Fig. 12. The filter exhibits quasi-elliptical characteristics with an excellent in-band insertion-loss of 0.4 $\mathrm{dB}$ centered at $6.85 \mathrm{GHz}$ with return-loss better than $10 \mathrm{~dB}$. The two transmission zeros near the upper and lower cut-off frequencies of $4.14 \mathrm{GHz}$ and $12.1 \mathrm{GHz}$, respectively, result in a filter with high selectivity. The out-of-band rejection is better than $20 \mathrm{~dB}$ over a very wideband between 2 to $3.59 \mathrm{GHz}$ and 12.2 to $20.74 \mathrm{GHz}$. The group-delay of the filter is approximately flat and is less than $0.1 \mathrm{~ns}$. The performance of the proposed UWB filter is compared in Table 1 with similar type of filters reported to date. 


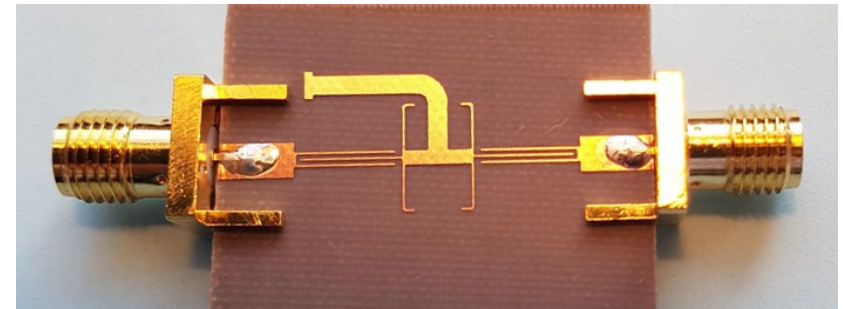

Fig. 11. The fabricated UWB BPF.

The proposed filter is constructed on a lower dielectric substrate however its size is comparable to others reported to date, as shown in Table 1. The UWB filter in references [8], [13] and [17] require short-circuit vias to ground. In references [10], [12], [14]-[16], and [18] the filter structure employs a defected ground plane which is located under the interdigital feed-lines. Unlike other UWB planar filters the proposed structure does not require any short-circuit via-holes and defected ground plane. This is important to reduce the complexity and manufacturing cost.


Fig. 12. Measured insertion-loss and return-loss response of the UWB BPF

(a) close-up view

(b) wideband view

\section{Conclusion}

Synthesis of a compact planar microstrip UWB bandpass filter is implemented using a novel multimode resonator structure that is via-free and requires no defected ground plane. The filter is shown to possess desirable characteristics of (i) sharp quasi-elliptical response; (ii) low passband insertion-loss; and (iii) super wide out-of-band rejection. These characteristics are normally achieved with high temperature superconductors. The multimode resonator employed is basically based on stepped impedance transmission-line resonator loaded with folded open-circuited stubs. The proposed structure excites three even and two oddmode resonances to realize the required UWB response.

Table 1: Comparison of the proposed UWB filter with recent publications

\begin{tabular}{|c|c|c|c|c|c|}
\hline Ref. & $\begin{array}{c}\text { Upper stopband } \\
(\mathrm{GHz}) \\
\text { for } \mathrm{S}_{21} \geq 20 \mathrm{~dB}\end{array}$ & $\begin{array}{c}\mathrm{S}_{21}(\mathrm{~dB}) \\
@ f_{\mathrm{o}}\end{array}$ & $\begin{array}{c}\mathrm{S}_{22}(\mathrm{~dB}) \\
@ f_{\mathrm{o}}\end{array}$ & $\begin{array}{c}\text { Size } \\
\lambda_{\mathrm{o}} \times \lambda_{\mathrm{o}}\end{array}$ & $\mathcal{E}_{r}$ \\
\hline$[8]$ & 12.6 & 3 & $>10$ & $0.44 \times 0.33$ & 2.45 \\
\hline$[9]$ & 7 & 2 & $>10$ & $0.40 \times 0.23$ & 3.5 \\
\hline$[10]$ & 17.6 & 2.5 & $>10$ & $0.38 \times 0.31$ & 2.55 \\
\hline$[11]$ & 14 & 1.3 & $>10$ & $0.34 \times 0.074$ & 9.8 \\
\hline$[12]$ & 18 & 1.5 & $>10$ & $0.51 \times 0.33$ & 2.55 \\
\hline$[13]$ & 27.6 & 1.5 & $>10$ & $0.51 \times 0.31$ & 2.55 \\
\hline$[14]$ & 28 & 1.8 & $>7$ & $1.10 \times 0.13$ & 2.2 \\
\hline$[15]$ & 29.7 & 1.4 & $>10$ & $0.51 \times 0.37$ & 2.55 \\
\hline$[16]$ & 30 & 2 & $>10$ & $0.52 \times 0.33$ & 2.55 \\
\hline$[17]$ & 14.5 & 0.9 & $>10$ & $0.70 \times 0.47$ & 2.2 \\
\hline$[18]$ & 17.5 & 0.8 & $>10$ & $0.60 \times 0.60$ & 3.55 \\
\hline$[19]$ & 14 & 1.3 & $>8$ & $0.23 \times 0.35$ & 6.15 \\
\hline$[20]$ & 15 & 3 & $>10$ & $0.62 \times 0.28$ & 6.15 \\
\hline This work & 35.5 & 0.4 & $>10$ & $0.46 \times 0.16$ & 2.17 \\
\hline
\end{tabular}

\section{References}

[1] "FCC, Revision of Part 15 of the Commission's Rules Regarding Ultra-Wideband Transmission Systems," Washington, DC, Tech. Rep, ET-Docket 98-153, FCC0248, Feb. 2002.

[2] S. Khalid, W. P. Wen, "Analysis of ultra-wide bandpass filter," IEEE National Postgraduate Conf., 2011, pp. 1- 4.

[3] K. D. Xu, Z. Luo, Y. Liu, Q. H. Liu, "High-selectivity single-ended and balanced bandpass filters using ring resonators and coupled lines loaded with multiple stubs," AEÜ Int. Journal of Electronics and Communications, 2018, 96, pp.193-198.

[4] T. Khorand, M. S. Bayati, "Novel half-mode substrate integrated waveguide bandpass filters using semihexagonal resonators," AEÜ Int. Journal of Electronics and Communications, 2018, 95, pp.52-58.

[5] Y. Yuan, S. Song Yang, R. Liu, "A Compact ultrawideband bandpass filter design based on open stub loaded SIR structure," 15th International Conference on Electronic Packaging Technology (ICEPT), 2014, pp. 1341-1343.

[6] Z. C. Hao, J. S. Hong, "Ultra-wideband filter technologies," IEEE Microw. Mag., 2010, 11, (4), pp. 5668.

[7] L. Zhu, S. Sun, W. Menzel, "Ultra-wideband (UWB) bandpass filter using multiple-mode resonator," IEEE Microw. Wirel. Compon. Lett., 2005, 15, (11), pp. 796798. 
[8] L. Xiuping, X. Ji, "Novel compact UWB bandpass filters design with cross-coupling between $\lambda / 4$ short-circuited stubs," IEEE Microwave and Wireless Components Letters, 2014, 24, (1), pp. 23-25.

[9] K. Rabbi, D. Budimir, "Highly selective reconfigurable filter for UWB systems," IEEE Microwave and Wireless Components Letters, 2014, 24, (3), pp. 146-148.

[10] A. Taibi, M. Trabelsi, A. Salimane, M. T. Belaroussi, J.-P. Raskin, "A Novel design method for compact UWB bandpass filters," IEEE Microwave and Wireless Components Letters, 2015, 25, (1), pp. 4-6.

[11]Z. Shang, X. Guo, B. Cao, B. Wei, X. Zhang, Y. Heng, G. Suo, X. Song, "Design of a superconducting ultrawideband bandpass filter with sharp rejection skirts and miniaturized size," IEEE Microwave and Wireless Components Letters, 2013, 23, (2), pp. 72-74.

[12]H. Zhu, Q.-X. Chu, "Compact ultra-wideband bandpass filter using dual-stub-loaded resonator (DSLR)," IEEE Microwave and Wireless Components Letters, 2013, 23, (10), pp. 527-529.

[13]H. Zhu and Q-X. Chu, "Ultra-wideband bandpass filter with a notch-band using stub-loaded ring resonator," IEEE Microwave and Wireless Components Letters, 2013, 23, (7), pp. 341-343.

[14] A. S. Hian Kuek, H. T. Su, M. K. Haldar, "Sextuple-mode ultra-wideband bandpass filter with wide upper stopband," 2014 IEEE Region 10 Symposium, pp. 1-5.

[15]X-H. Wu, Q-X. Chu, X-K. Tian, X. Ouyang, "Quintuplemode UWB bandpass filter with sharp roll-off and superwide upper stopband," IEEE Microwave and Wireless Components Letters, 2011, 21, (12), pp. 661-663.

[16]H. Zhu and Q-X. Chu, "Ultra-wideband (UWB) bandpass filter with sharp selectivity and wide upper stopband," IEEE Int. Conference on Ultra-Wideband, 2012, pp. $103-105$.

[17] A. Kamma et al., "Multi mode resonators based triple band notch UWB filter," IEEE Microwave and Wireless Components Letters, 2017, 27, (2), pp. 120-122.

[18] B. Mohammadi, A. Valizade, J. Nourinia, P. Rezaei, "Design of a compact dual-band-notch ultra-wideband bandpass filter based on wave cancellation method," IET Microw. Antennas Propag., 2015, 9, (1), pp. 1-9.

[19] Y. Li, W. Li, W. Yu, C. Liu, "A miniaturization band-pass filter with ultra-narrow multi notch-band characteristic for ultra-wideband communication applications," ACES Journal, 2014, 29(4), pp. 289-300.

[20] Y. Li, W. Li, C. Liu, Q. Ye, "A compact UWB band-pass filter with ultra-narrow tri-notch band characteristic," ACES Journal, 2014, 29(2), pp. 170-177.

[21]Q.-X. Chu, X.-K. Tian, "Design of UWB bandpass filter using stepped-impedance stub-loaded resonator," IEEE Microwave and Wireless Components Letters, 2010, 20, (9), pp. 501-503.

[22] C.-W. Tang and Y.-K. Hsu, "A Microstrip Bandpass Filter With Ultra-Wide Stopband," IEEE Trans. Microwave Theory \& Techniques, 2008, 5, (6), pp. 1468-1472. 